\title{
Fast-ion transport and neutral beam current drive in ASDEX Upgrade
}

\author{
B Geiger ${ }^{1}$, M Weiland ${ }^{1}$, A S Jacobsen², D Rittich ${ }^{1}, \mathbf{R}$ Dux $^{1}, \mathbf{R}$ \\ Fischer $^{1}$, C Hopf ${ }^{1}$, M Maraschek ${ }^{1}$, R M McDermott ${ }^{1}$, S K \\ Nielsen $^{2}$, T Odstrcil ${ }^{1}$, M Reich ${ }^{1}$, F Ryter ${ }^{1}$, M Salewski $^{2}$, P A \\ Schneider $^{1}$, G Tardini $^{1}$, the ASDEX Upgrade team ${ }^{1}$ \\ 1 Max-Planck-Institute for Plasma Physics, Boltzmannstr. 2, 85748 Garching, \\ Germany \\ 2 Technical University of Denmark, Department of Physics, Dk-2800 Kgs. Lyngby, \\ Denmark
}

PACS numbers: 52.55.Fa, 52.50.Gj, 52.55.Tn, 52.55.Wq

\begin{abstract}
.
The neutral beam current drive efficiency has been investigated in the ASDEX Upgrade tokamak by replacing on-axis neutral beams with tangential off-axis beams. A clear modification of the radial fast-ion profiles is observed with a fast-ion Dalpha diagnostic that measures centrally peaked profiles during on-axis injection and outwards shifted profiles during off-axis injection. Due to this change of the fast-ion population, a clear modification of the plasma current profile is predicted but not observed by a motional Stark effect diagnostic.

The fast-ion transport caused by MHD activity has been studied in low collisionality discharges that exhibit strong $(1,1)$ modes. In particular due to sawtooth crashes, significant radial redistribution of co-rotating fast-ions is observed which can very well be described by the Kadomtsev model. In addition, first tomographic reconstructions of the central 2D fast-ion velocity space in the presence of sawtooth crashes allow the investigation of the pitch dependence of the mode-imposed redistribution: A stronger redistribution of mainly co-rotating fast ions is observed than of those with smaller pitch values.
\end{abstract}




\section{Introduction}

In present day fusion devices fast ions are mainly generated by neutral beam injection (NBI). They heat plasmas through collisions with electrons and ions and, if injected with a sufficiently parallel velocity component, their associated current drive can be used to reach non-inductive conditions or to tailor the plasma current profile [1]. Well confined fast ions are, hence, essential to obtain good heating and current drive performances. In addition, detailed understanding of the fast-ion transport mechanisms is needed in view of future fusion devices, not only to guarantee good performance, but also to ensure the safety of the machine: fast helium ions will be produced in fusion reactions that could, if poorly confined, damage the first wall [2]. In toroidally axisymmetric devices such as tokamaks, the fast-ion transport induced by collisions and orbit effects, i.e. the neo-classical transport, is relatively small. However, enhanced fast-ion transport, caused by large- and small-scale instabilities (anomalous transport), could reduce the fast-ion confinement and must, therefore, be investigated. In particular, core localized MHD modes must be studied in detail because they spatially overlap with the fast-ion distribution functions which are typically peaked on-axis. One of the most severe types of core-perturbation in tokamak plasmas is induced by sawtooth crashes [3] which are caused by the fast reconnection of a mode with toroidal and poloidal mode numbers of one $(n=1$ and $m=1)$. They appear periodically in the plasma center when the safety factor, $\mathrm{q}$, is below one and cause a fast crash of the central pressure, followed by a recovery phase. The crashes strongly redistribute fast ions, as reported from DT experiments at JET [4] and TFTR [5] and from deuterium plasmas at DIII-D [6], TEXTOR [7], MAST [8] and ASDEX Upgrade [9]. There are two mechanisms that are responsible for the strong redistribution. These are $\mathbf{E x B}$ drift effects and the motion of the fast particles along the evolving magnetic field lines. The details of these mechanisms are, however, not yet clear and need to be investigated in detail.

Of special interest is also the characterization of the off-axis neutral beam current drive (NBCD) efficiency, which is motivated by somewhat contradicting results in the past. On the one hand, previous experiments in ASDEX Upgrade showed that the evolution of the current profile did not follow neo-classical predictions when replacing onaxis NBI with off-axis NBI. In particular, the effect of off-axis NBCD on measurements of the current profile by a motional Stark effect diagnostic (MSE) was weaker than predicted by theory [10]. On the other hand, current-drive studies based on vertically shifted plasmas at DIII-D [11] agreed with modeling. Moreover, measured radial fast- 

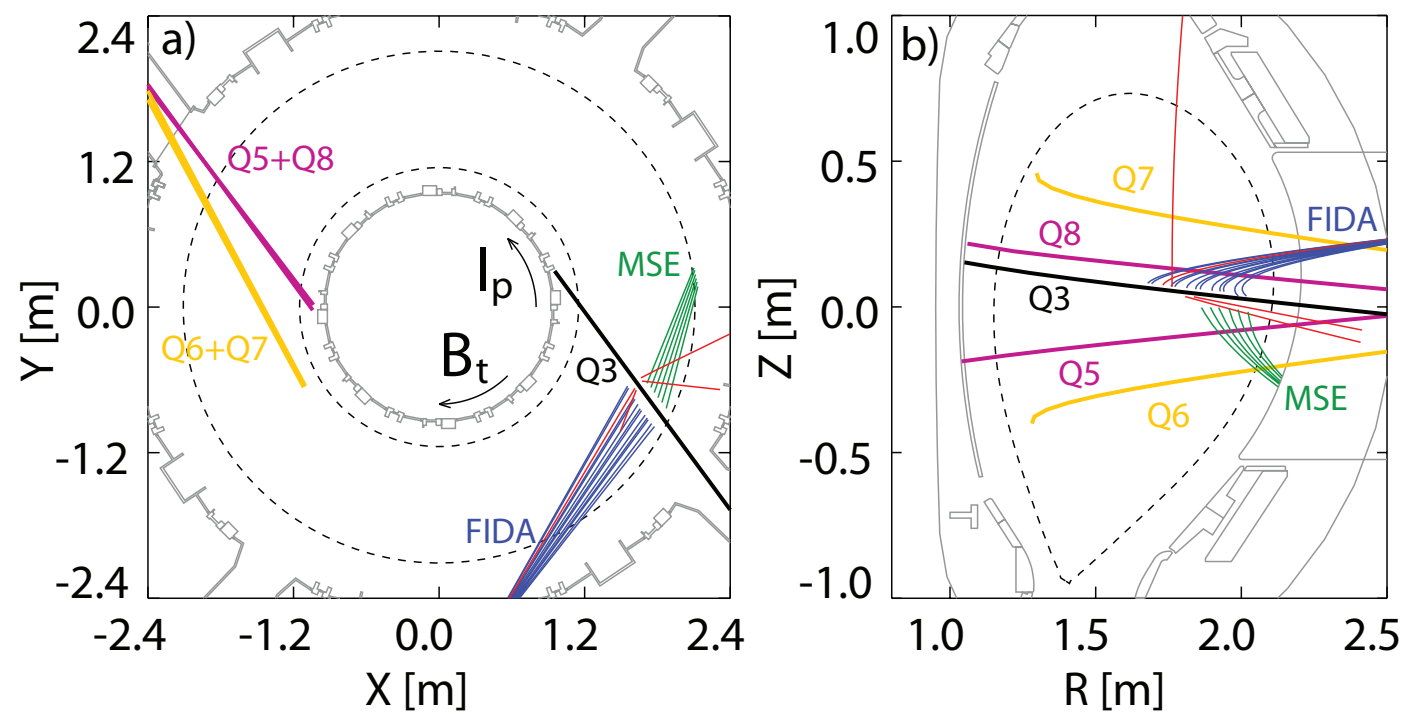

Figure 1. Top down and poloidal view of the ASDEX Upgrade tokamak. The geometry of the off-axis NBI sources is sketched in yellow and that of the on-axis sources applied during the experiments is illustrated in purple (93 keV) and black (60 $\mathrm{keV}$ ). The toroidal lines of sight of the FIDA diagnostic are shown in blue and those used for a tomographic reconstruction of the central fast-ion velocity space distribution are indicated in red. The viewing geometry of the MSE diagnostic is plotted in green.

ion profiles, which are expected to be linked to the beam driven current, exhibited good agreement with neo-classical predictions $[12,13]$.

This important aspect, as well as the fast-ion redistribution due to sawtooth crashes, has now been revisited in ASDEX Upgrade [14], motivated by new measurement capabilities. In particular, a fast-ion D-alpha (FIDA[15]) spectroscopy diagnostic is now available that measures radial profiles of co-rotating fast-ions and allows reconstructions of central 2D fast-ion velocity distribution functions by a tomographic inversion in velocity space.

This paper is structured as follows. In section II, a study of off-axis fast-ion populations and the associated NBI current drive is presented. Radial fast-ion profiles and MSE measurements are compared with theoretical predictions. The effect of sawtooth crashes on the fast ions is discussed in section III. Measured neutron rates and radial FIDA profiles of co-rotating ions are compared with theoretical predictions and the central fast-ion velocity space is analyzed using tomographic inversions. Finally, a short summary and conclusion are given. 


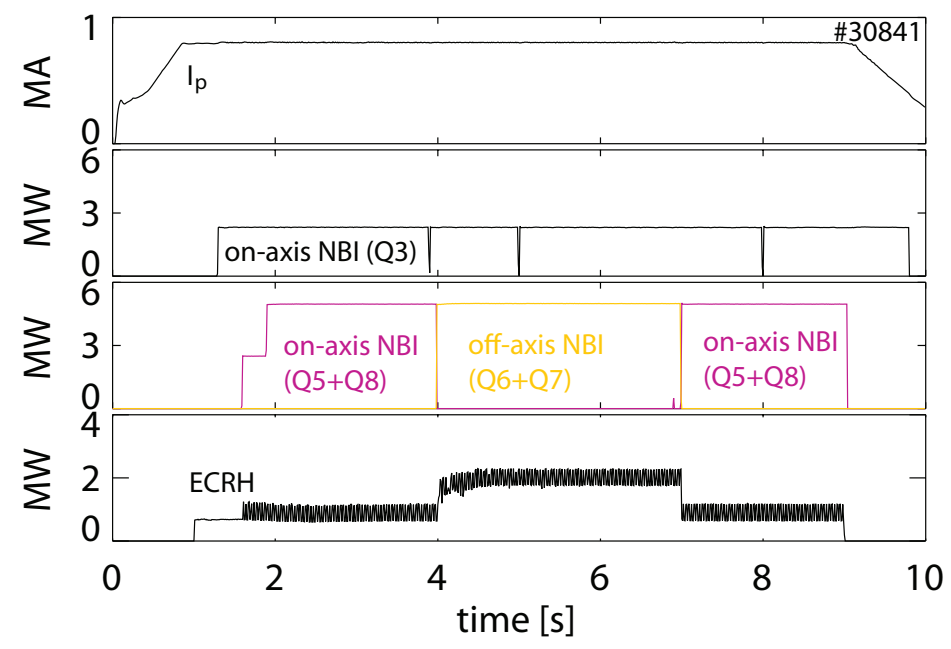

Figure 2. Time traces of discharge \#30841 showing the evolution of the plasma current, the NBI heating power and the ECRH heating power.

\section{Fast-ion current drive study}

The ASDEX Upgrade tokamak is very well equipped for neutral beam current drive studies because it has a sensitive set of fast-ion diagnostics, an MSE diagnostic and flexible heating and current drive systems. Up to $5 \mathrm{MW}$ of electron cyclotron resonance heating $(\mathrm{ECRH})$ and current drive (ECCD) are available to maintain constant electron temperatures (feedback control of $T_{e}$ ) and to stabilize MHD instabilities $[16,17]$. Moreover, eight neutral beam sources with each 2.5 MW of heating power are installed of which two beams have an off-axis geometry. In figure 1, the geometry of those NBI sources applied during this study is displayed. The off-axis beams, illustrated in yellow (Q6+Q7), inject above and below the plasma center and have a tangential geometry.

The fast-ion current drive efficiency of the off-axis sources has been investigated in discharges with a toroidal magnetic field of $-2.6 \mathrm{~T}$ and a feedback controlled plasma current of 0.8 MA. An overview plot of the heating scheme applied in a representative discharge (\#30841) is shown in figure 2. Starting at $1.3 \mathrm{~s}, 2.5 \mathrm{MW}$ of heating power from the $60 \mathrm{keV}$ on-axis source Q3 was applied because this source is needed for charge exchange recombination spectroscopy (CXRS) [18], MSE and FIDA measurements. From about $1.7 \mathrm{~s}$ on, additional $5 \mathrm{MW}$ of heating power were injected by the two on-axis neutral beams which are indicated in purple in figure 1 . Between $4 \mathrm{~s}$ and $7 \mathrm{~s}$, these two radial sources were replaced by the tangential off-axis beams.

In addition to the NBI heating power, up to $2 \mathrm{MW}$ of ECRH were applied to avoid sawtooth crashes (co-ECCD inside $q=1)$, to avoid the formation of a $(2,1)$ magnetic 


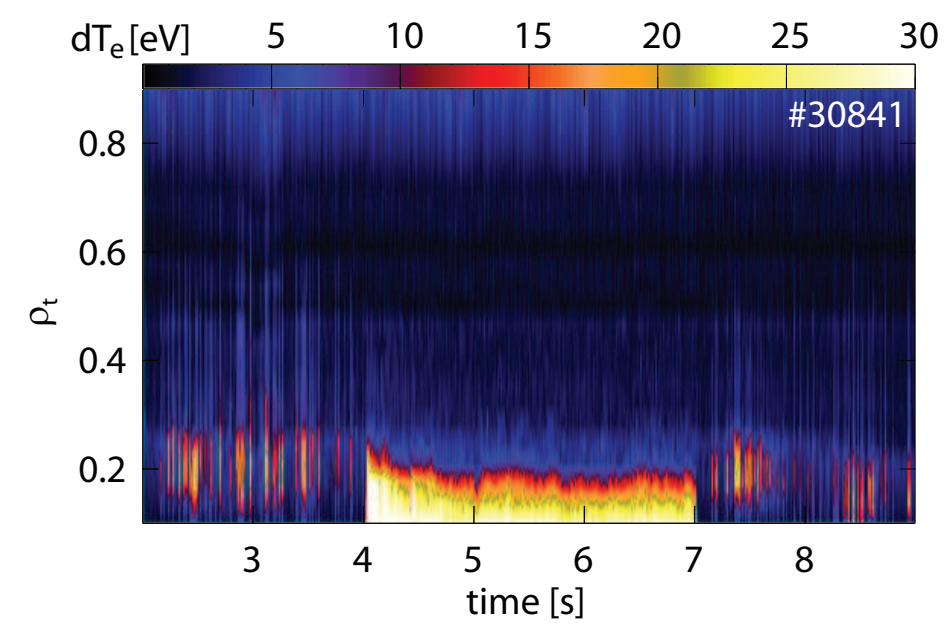

Figure 3. Evolution of the maximum $T_{e}$ perturbation amplitude as a function of the toroidal flux label for frequencies between $5 \mathrm{kHz}$ and $30 \mathrm{kHz}$. During on-axis NBI, fishbone modes are present and a continuous $(1,1)$ mode is localized in the plasma core during off-axis NBI.

island (modulated co-ECCD at the $\mathrm{q}=2$ surface) and to prevent the drop of the central electron temperature during off-axis NBI. These three aims were achieved in discharge \#30841. The central electron temperature did not drop during the off-axis NBI phase, no sawtooth crashes and no $(2,1)$ magnetic islands were observed. Only core localized $\mathrm{n}=1, \mathrm{~m}=1$ modes in the frequency range between $10 \mathrm{kHz}$ and $25 \mathrm{kHz}$ were present. The positions of these modes were determined by studying the mode-induced electron temperature fluctuations that are measured by an electron cyclotron emission (ECE) diagnostic. In figure 3, the evolution of the amplitude of the dominant mode is plotted as a function of the normalized toroidal flux coordinate, $\rho_{\mathrm{t}}$. During on-axis NBI, $(1,1)$ modes, identified as fishbones [19], are located at $\rho_{\mathrm{t}} \approx 0.2$ and during off-axis NBI, a continuous mode is localized at $\rho_{\mathrm{t}} \approx 0.15$.

Figure 4 shows the evolution of electron and ion temperature profiles, as well as of profiles of the electron density and plasma rotation frequency. The electron density (inferred by integrated data analysis (IDA) [20]) remains constant during the experiment while the central ion temperature and rotation (both measured by CXRS diagnostics) drop during the off-axis NBI phase, which is well explained by reduced central heating and momentum input. The impurity density, measured by CXRS (on helium, boron and nitrogen), remained low and constant during the experiments and result in an effective charge number of about 1.3, which is agreement with the measured level of Bremsstrahlung. As mentioned above, we tried to keep the electron temperature constant during the experiment by adding $\approx 0.8 \mathrm{MW}$ of feedback controlled central 

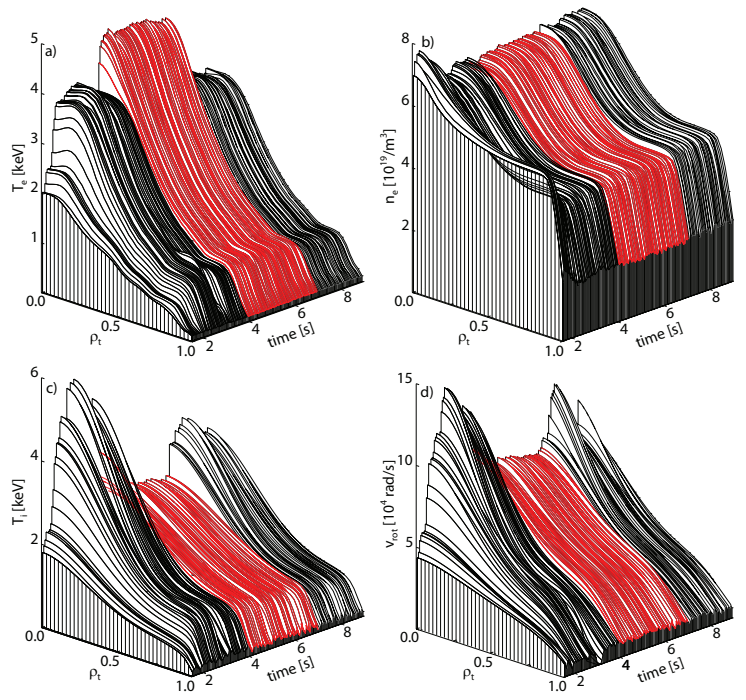

Figure 4. Bulk plasma profiles of discharge \#30841 showing the electron temperature (a), the electron density (b), the ion temperature (c) and the toroidal plasma rotation frequency (d). The profiles acquired during off-axis NBI are plotted in red.
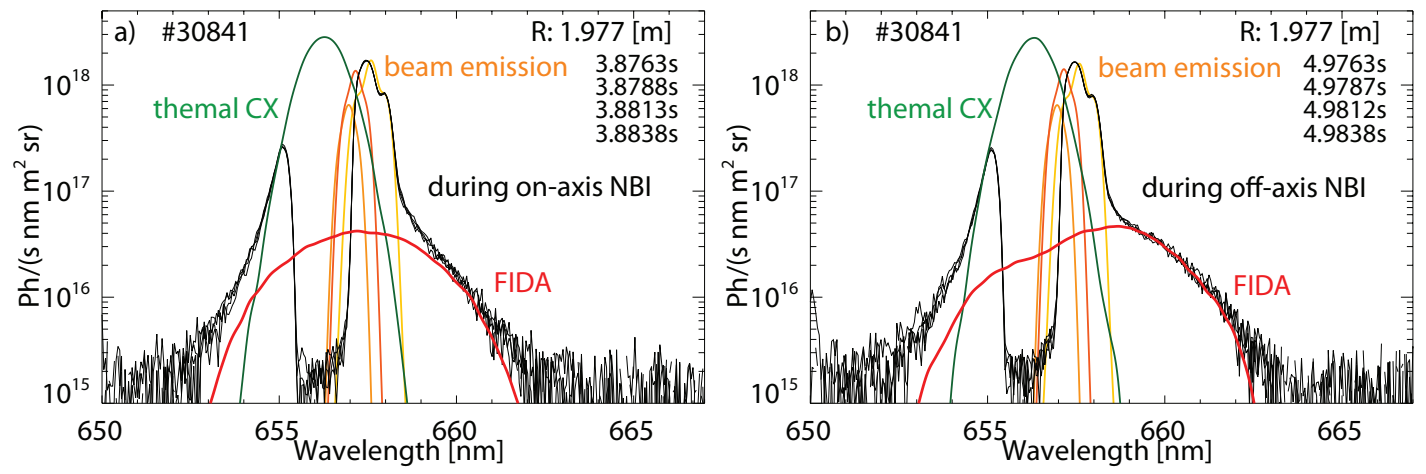

Figure 5. Measured spectra at $\rho_{\mathrm{t}} \approx 0.55$ during on-axis (a) and off-axis (b) NBI heating. In color, synthetic spectra from FIDASIM are plotted.

ECRH power between $4 \mathrm{~s}$ and $7 \mathrm{~s}$. Constant electron temperatures are necessary for NBI current drive studies because the shape of the ohmic current profile depends strongly on $\mathrm{T}_{\mathrm{e}}$ and significant changes would make the analysis of the off-axis neutral beam current drive efficiency very difficult. Here, the electron temperature is slightly too high in the plasma center during the off-axis phase. This is not ideal but can be taken into account by the forward modeling.

The fast-ion population during on-axis NBI and off-axis NBI has been studied by a FIDA spectroscopy diagnostic [21] which analyzes Doppler-shifted Balmer alpha radiation $\left(\lambda_{0}=656.1 \mathrm{~nm}\right)$ from fast ions that undergo charge exchange reactions along the path of NBI Q3. 
Example spectra measured by a line of sight that intersects NBI Q3 at $\rho_{\mathrm{t}} \approx 0.55$ are shown in figure 5 during on-axis NBI and during off-axis NBI. The spectra, plotted on a semi-logarithmic scale, have been measured with a temporal resolution of $2.5 \mathrm{~ms}$ and passive radiation, acquired during a $10 \mathrm{~ms}$ long phase without operation of NBI Q3, has been subtracted. Between 655 and $657 \mathrm{~nm}$, a filter blocks un-shifted Balmer alpha radiation from the plasma edge to avoid saturation effects. The spectral wing above $659 \mathrm{~nm}$ corresponds to the charge exchange radiation of fast-ions (FIDA radiation), which is more pronounced during off-axis NBI than during on-axis NBI. This is expected because off-axis NBI generates a larger fast-ion population at the measurement position $\left(\rho_{\mathrm{t}} \approx 0.55\right)$ than on-axis NBI. In addition to the measurement, simulated spectra are shown in color. The spectra have been calculated by the synthetic diagnostic FIDASIM [22] which needs to be supplied with theoretical fast-ion distribution functions. For the study presented here, the theoretical fast-ion distribution functions are predicted by the TRANSP code [23]. TRANSP needs information on kinetic profiles, the heating sources, the initial q-profile, information on the separatrix position, the toroidal magnetic field and the plasma current as inputs and uses a the Monte Carlo module NUBEAM [24] to simulate fast ions. The code is based on neo-classical theory, but one can also add a predefined diffusive like fast-ion transport. The simulated FIDA radiation, plotted in red in figures $5 \mathrm{a}$ and $5 \mathrm{~b}$, agrees well with the red-shifted wing of the measured radiation. This already suggests that TRANSP consistently describes the fast-ion distribution function during on-axis NBI and off-axis NBI. In addition to the simulated FIDA radiation, the predicted beam emission (yellow/orange) and the thermal charge exchange component (green), i.e. the halo radiation, are shown. The good agreement between these components and the measurement is an important consistency check when comparing FIDA measurement and simulation.

Information on radial fast-ion profiles is obtained by analyzing the spectra from an array of lines of sight that intersect the NBI path at different positions. Depending on the viewing geometry relative to the magnetic field direction and on the observed wavelength range, different parts of the fast-ion velocity space can be sampled (see [13]). Here, we use toroidal lines of sight (shown in blue in figure 1) and focus on red shifted radiation between 660 - $661 \mathrm{~nm}$. This corresponds to co-rotating fast-ions with energies above $30 \mathrm{keV}$ which cover the part of the fast-ion velocity space distribution that contributes most to the fast-ion current. Radial profiles of the corresponding FIDA radiation are displayed in figure 6. Passive radiation, measured in phases without operation of NBI Q3, has been subtracted from the spectra before integrating. Moreover, the data has 

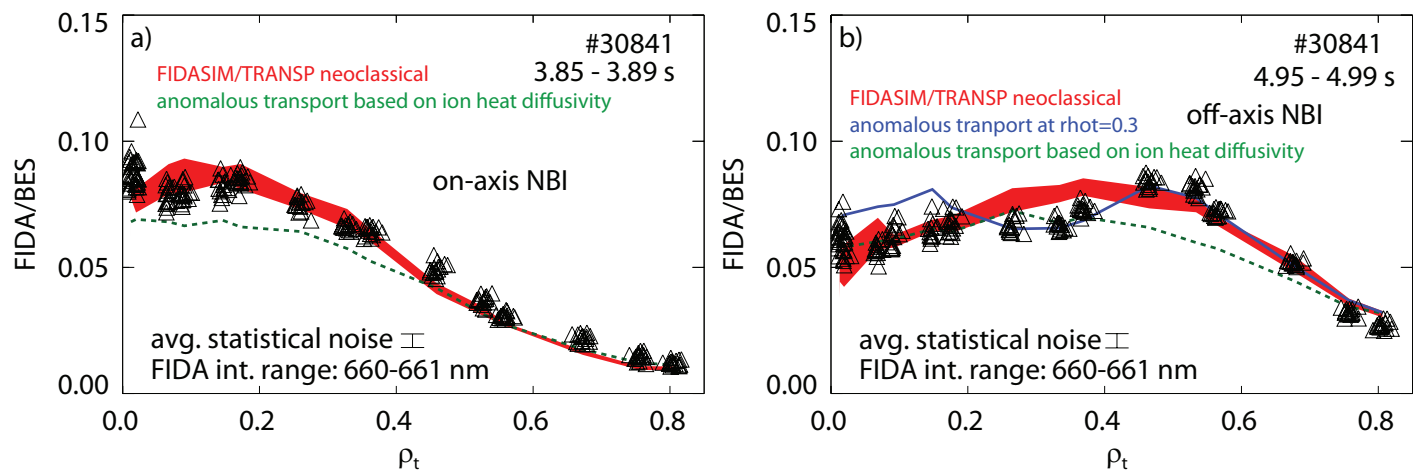

Figure 6. Radial FIDA/BES profiles during on-axis NBI (a) and off-axis NBI (b) compared with predictions by TRANSP+FIDASIM. In red, a sensitivity scan of neoclassical predictions is shown that is based on the variation of the kinetic profiles input to TRANSP (plus and minus one sigma of the measurement uncertainties of $\left.\mathrm{T}_{\mathrm{e}}, \mathrm{T}_{\mathrm{i}}, \mathrm{n}_{\mathrm{e}}, \mathrm{Z}_{\mathrm{eff}}, \mathrm{v}_{\text {tor }}\right)$. In blue, the TRANSP+FIDASIM predictions is illustrated that represent a local anomalous fast-ion diffusivity during off-axis NBI at $\rho_{\mathrm{t}}=0.3$. The simulated FIDA/BES profiles in green correspond to anomalous transport based on the calculated ion-heat diffusivity (scaled by 0.1 ).

been normalized by the simultaneously measured beam emission (BES) which enables the neutral beam attenuation to be taken into account and to be independent of the intensity calibration.

The measured radial FIDA/BES profiles show a clear modification of the fast-ion population when switching from on-axis NBI (see figure 6a) to off-axis NBI (see figure $6 \mathrm{~b})$. Furthermore, the measured profiles are in good agreement with the prediction from TRANSP+FIDASIM. The profiles shown in red have been calculated by integrating the simulated spectra in wavelength and applying the normalization based on the simulated beam emission. The widths of the synthetic profiles correspond to \pm one standard deviation of the uncertainties of predictions. The latter have been determined by a sensitivity scan using ten different TRANSP and FIDASIM runs per time point. For each run, one of the kinetic profiles $\left(\mathrm{T}_{\mathrm{e}}, \mathrm{T}_{\mathrm{i}}, \mathrm{n}_{\mathrm{e}}, \mathrm{Z}_{\mathrm{eff}}, \mathrm{v}_{\text {tor }}\right)$, input to TRANSP and FIDASIM, was increased or decreased by one standard deviation of the corresponding statistical measurement uncertainties. The resulting differences relative to the nonmodified prediction have been summed up by applying a Gaussian error propagation.

During on-axis NBI (figure 6a), the simulated profiles agree very well with the simulation. Also during off-axis NBI, simulation and measurement roughly match. However, the prediction slightly overestimates the measured FIDA/BES signal between $\rho_{\mathrm{t}}=0.2-0.4$. By assuming an additional anomalous fast-ion diffusivity in TRANSP 


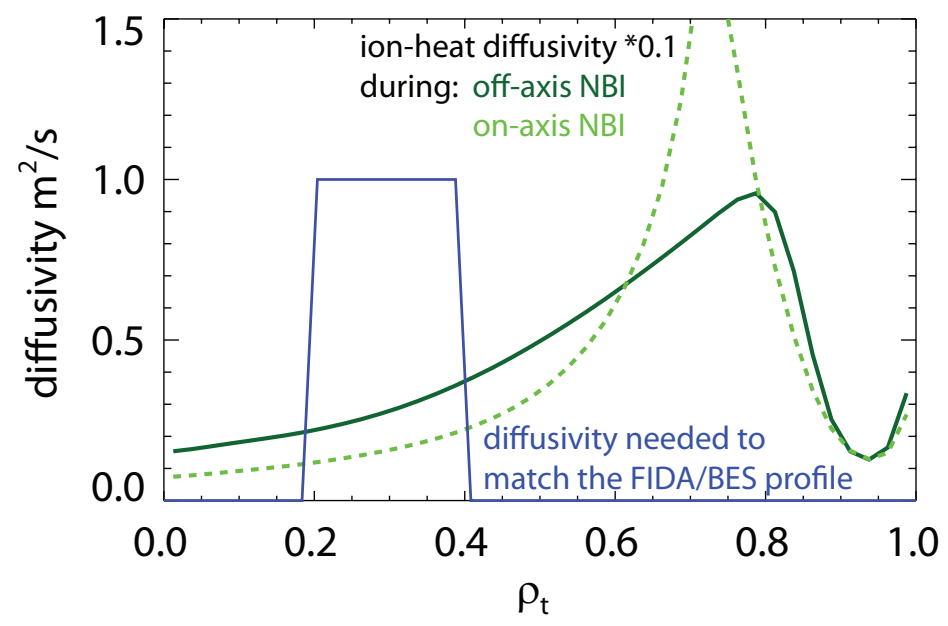

Figure 7. Radial profiles of the different anomalous fast-ion diffusion coefficient input to TRANSP.

between $4 \mathrm{~s}$ and $7 \mathrm{~s}$ that is localized at $\rho_{\mathrm{t}} \approx 0.3$ (see details in figure 7 ) the simulation agrees better with the experimental data, as plotted in blue in figure $6 \mathrm{~b}$.

The origin of this localized anomalous fast-ion diffusivity, which is needed to explain the measurement, is not clear yet. The geometry of the NBI system has been checked with dedicated beam-into-gas experiments which makes a geometry induced discrepancy very unlikely. The continuous $(1,1)$ mode present in discharge \#30841 does not obviously cause an anomalous fast-ion diffusivity at $\rho_{\mathrm{t}}=0.3$ because it is located at $\rho_{\mathrm{t}}=0.15$. Anomalous fast-ion diffusivity induced by turbulence is also unlikely because, based on a power balance analysis, we would expect the strongest effect of turbulent transport further out. Figure 7 shows radial profiles of the TRANSP-calculated ion heat diffusivity $\left(D_{\text {fast }}=0.1 * \chi_{\text {ion-heat }}\right)$ during on-axis NBI and during off-axis NBI. The maximum of the heat diffusivity, which indicates the location of strong turbulent transport, is clearly not located at $\rho_{\mathrm{t}}=0.3$ but at $\rho_{\mathrm{t}}=0.7$. To test the possibility of turbulence induced fastion diffusivity, the scaled ion heat diffusivity has been input to a TRANSP+FIDASIM simulation. However, the resulting FIDA/BES profiles shown in figure 6 in green clearly do not agree with the measurement.

Therefore, we unfortunately cannot resolve the discrepancy between the FIDA measurement and the simulation. However, being able to simulate a fast-ion population that matches the experimental data, we can investigate the current drive efficiency of this fast-ion distribution function.

To calculate the current distribution, several options are available in TRANSP. For the study presented here, we applied the Sauter formula [25] to calculate the plasma 


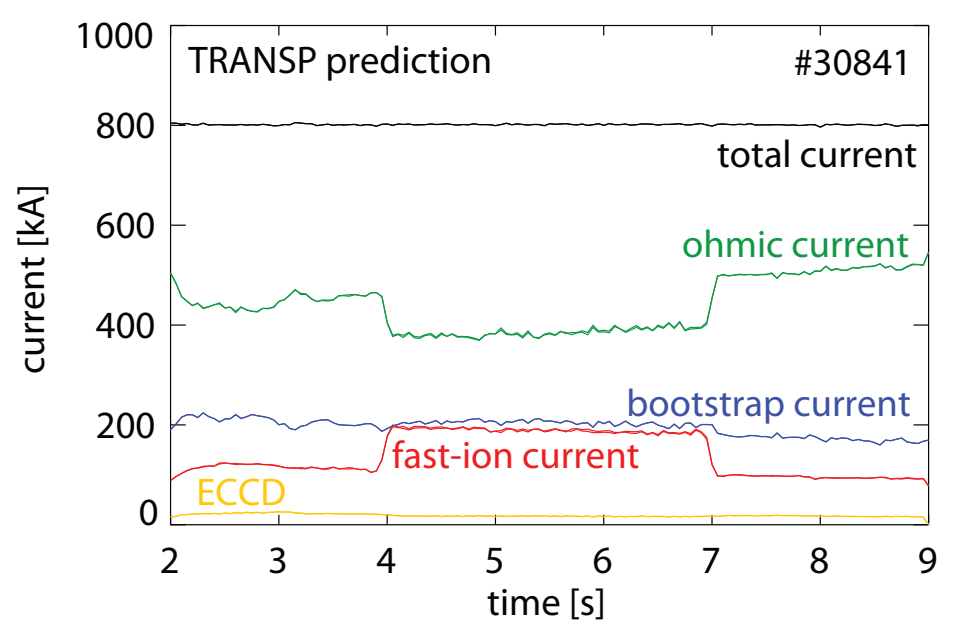

Figure 8. TRANSP predicted composition of the plasma current when assuming neoclassical fast-ion transport and when assuming a localized level of anomalous fast-ion diffusivity during off-axis NBI (there is almost no difference between the two cases).

resistivity and the bootstrap current, TORAY [26] to determine the current driven by ECCD and the Lin-Liu formula [27] to calculate the shielding of the positive current carried by the fast ions.

In figure 8, the TRANSP predicted evolution of the contributions to the plasma current are illustrated. In red, the fast-ion current is shown. It increases between $4 \mathrm{~s}$ and $7 \mathrm{~s}$, well explained by the tangential injection geometry of the off-axis NBI sources. The increased fast-ion current is immediately balanced by a reduction of the feedback controlled ohmic current while the contributions of the bootstrap current and the current driven by ECCD remain constant. In addition to this neo-classical simulation, the current expected when applying the artificial fast-ion diffusion coefficient at $\rho_{\mathrm{t}}=0.3$ during off-axis NBI is shown. However, the second curve can hardly be seen because the current drive efficiency is almost unchanged. It should be noted that assuming anomalous transport in the simulation does not significantly change the total current driven by off-axis NBI because it mainly broadens the fast-ion profile. The reduced current drive efficiency of fast ions that are redistributed outwards (e.g. lost or slowed down more quickly) is balanced by an inward diffusion of fast ions where the increased slowing down time allows particles to contribute more to the plasma current.

Experimentally, a reduced ohmic current is observed during off-axis NBI since the loop voltage, induced by the ohmic transformer coil to drive the current, drops. As shown in figure 9 , the measured loop voltage is reduced by $\approx 50 \%$ between 4 and $7 \mathrm{~s}$. This observation is in good agreement with the TRANSP predicted loop voltage, shown 


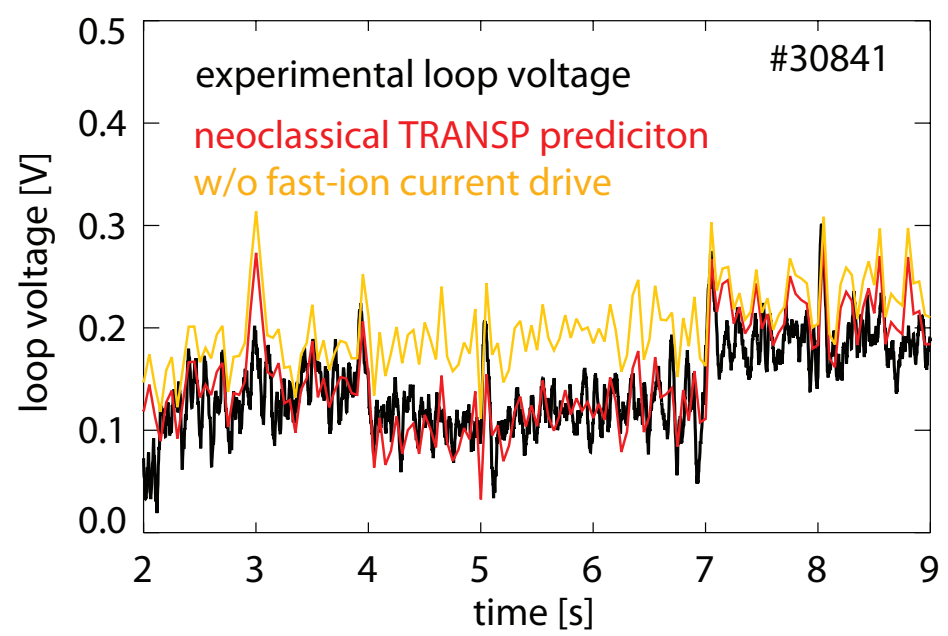

Figure 9. Measured loop voltage compared with predictions from TRANSP. The simulation plotted in red takes the current driven by the fast ions into account while the simulation given in yellow does not.
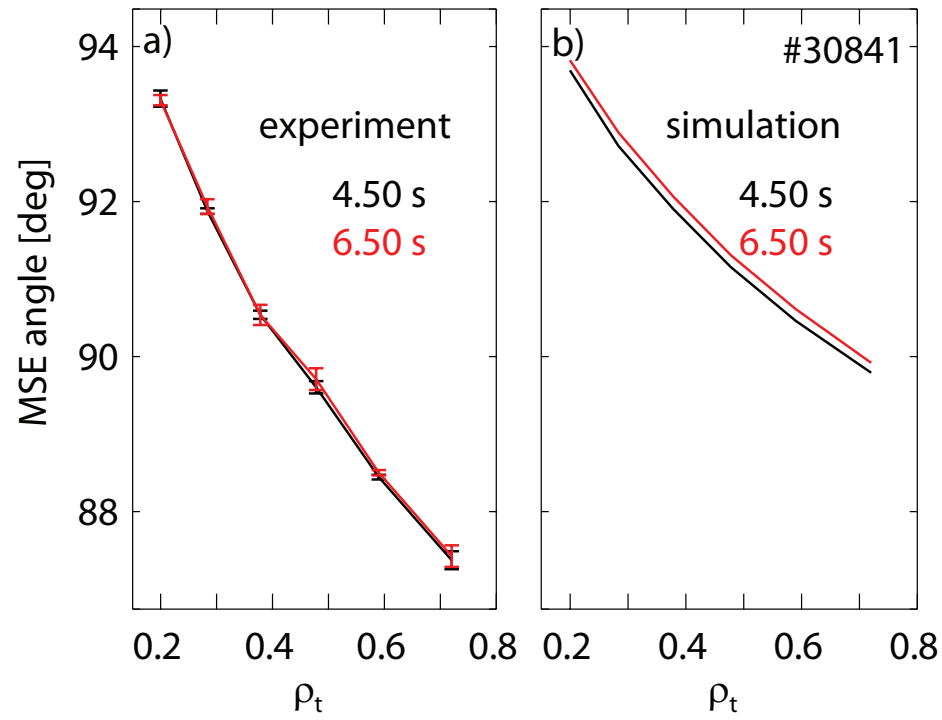

Figure 10. Measured (a) and TRANSP-predicted (b) MSE angles for time points shortly and $2.5 \mathrm{~s}$ after switching to off-axis NBI.

in red. In contrast, when assuming no current driven by the fast ions (simulation plotted in yellow) prediction and experiment disagree. This shows without ambiguity that the reduction of the loop voltage is mainly caused by an increased current-drive from the off-axis NBI. In addition, it should be noted that the good match between the predicted and measured loop-voltage also validates the kinetic profiles input to TRANSP.

In contrast to this good agreement with the neo-classical simulation, the comparison between measured and predicted MSE angles shows differences. Figure 10 compares 
radial profiles of the MSE measurement for two time points during the off-axis phase. The MSE diagnostic measures the projection of the polarization direction of the sigma component of the beam emission of $60 \mathrm{keV}$ neutrals of NBI Q3 on the plane perpendicular to its lines of sight (see geometry in figure 1). The polarization direction depends on the electric field vector encountered by the neutrals which are injected with the velocity $\mathbf{v}_{n}$. This electric field consists of the $\mathbf{v}_{n} \times \mathbf{B}$ Lorentz field and the radial electric field. The latter has been calculated based on the radial force balance [28] and on the assumption that the poloidal rotation velocity of deuterium is negligible (valid for neo-classical conditions). Here, it should be noted that our previous publication ([13]) neglected the diamagnetic term and applied the wrong sign for the radial electric field (a bug in TRANSP which has been resolved now). This overestimated of the change of the predicted MSE angles by about 20\% NBI but did not qualitatively modify the result.

The combination of the line of sight geometry of the MSE diagnostic and the radial injection geometry of NBI Q3 provides a good sensitivity of the MSE diagnostic on changes of the poloidal magnetic field and, hence, on changes of the plasma current profile. The predicted MSE profile, plotted in figure 10b, shows a clear modification between $4.5 \mathrm{~s}$ and $6.5 \mathrm{~s}$ (shortly and $2.5 \mathrm{~s}$ after switching to off-axis NBI). This change is expected during the off-axis phase because the current profile and, thus, the poloidal magnetic field, are supposed to vary slowly on the resistive time scale of seconds. First, the current generated by off-axis NBI is completely compensated by a reduction of the local ohmic current. Then, the current profile should change according to the off-axis beam deposition on the resistive time scale. However, even after $2.5 \mathrm{~s}$, the measured MSE profiles hardly exhibit any changes (see figure 10a).

Also when comparing the temporal evolution of the MSE data with the prediction by TRANSP, a disagreement is observed compared to the neo-classical simulation. Figure 11 shows time traces of the individual channels of the MSE diagnostic. A radial sweep of $\approx 2 \mathrm{~cm}$ of the plasma at $\approx 3$ s proves the reliability of the diagnostic through the clear change in the measurement that is well reproduced by TRANSP. Moreover, the diagnostic resolves well a step in the measured MSE angles at $4 \mathrm{~s}$ directly after the application of off-axis NBI. This is explained by a change of the Shafranov shift induced by a variation in the fast-ion pressure profile. To be able to compare the time evolution with the TRANSP prediction, a channel dependent offset has been added to the simulation to match the experimental data between 3 and $4 \mathrm{~s}$. As can be seen, the neo-classically predicted MSE angles in red change more than the measured ones. Also when assuming the level of anomalous fast-ion diffusivity at $\rho_{\mathrm{t}} \approx 0.3$ which is needed 


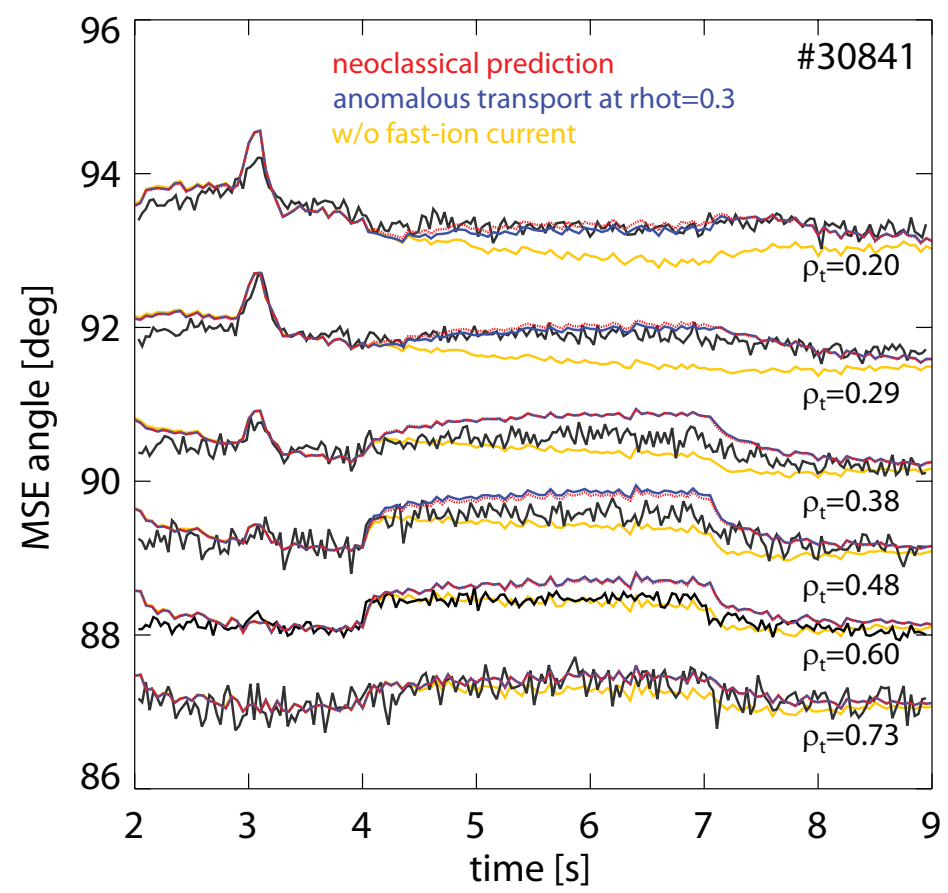

Figure 11. Temporal evolution of the measured and predicted MSE angles. In red, the neo-classical prediction by TRANSP is shown. The prediction in blue represents the application of a local anomalous fast-ion diffusivity during the off-axis NBI phase and the simulation in yellow represents no fast-ion driven current.

to match the FIDA measurements during off-axis NBI, a very similar result to the neo-classical prediction is obtained (see the blue curve in figure 11). In contrast, the simulation changes substantially when switching off the fast-ion-driven current in the simulation. Without fast-ion current drive, the predicted evolution of the MSE angles strongly differs from the measured one. This proves that the NBI current drive efficiency is not zero. However, the profile of the driven current differs from the prediction.

One possible explanation for the disagreement of the experimental and predicted current profile could be an incomplete model of the shielding factor of the positive ion current. However, all options available in the TRANSP code to calculate the fast-ion driven current show very similar results (e.g. the application of a collisionality dependent shielding factor ([29]) does not significantly change the NBI current drive efficiency.). We also tested if the choice of the initial q-profile in a TRANSP simulation changes the conclusion but this is not the case. Further, a sensitivity scan similar to figure 6 has been conducted. However, the modifications are very small and cannot be displayed properly. Another possibility to explain the data would be that the fast-ions behave neo-classically in the observed part of the velocity space but experience strong anomalous transport 
in other regions. Further, the $(1,1)$ mode activity during on-axis NBI could already broaden the plasma current distribution, which would make the effect of off-axis NBI less pronounced. These two possibilities need to be investigated in future experiments with q-profiles above one and, hence, without $(1,1)$ modes and with extended capabilities to diagnose the fast-ion distribution functions.

\section{Sawtooth-induced fast-ion transport}

In the 2014 experimental campaign of ASDEX Upgrade, fast-ion transport experiments were performed with $1 \mathrm{MA}$ of plasma current, a toroidal magnetic field of $-2.6 \mathrm{~T}$ and 2.5 MW of heating power from one radially injecting on-axis NBI source (NBI Q3). The discharges feature low densities $\left(\approx 4 \times 10^{19} / \mathrm{m}^{3}\right)$ and high ion temperatures (up to $6 \mathrm{keV}$ ) and exhibit strong sawtooth activity, indicated by periodic drops of the central electron temperature, ion temperature and rotation. The magnetic spectrogram in figure $12 \mathrm{a}$ shows the footprint of fishbone modes that appear prior to the sawtooth crashes. In addition, modes are visible after the crashes which indicates an incomplete magnetic reconnection during the crashes which is a routine phenomenon observed in ASDEX Upgrade [30]. The crash times of the sawteeth are in the range of $0.1 \mathrm{~ms}$ which can be determined from tomographic reconstructions of soft X-ray measurements. Figure 12c shows the position of the radiating plasma center as a function of time for one sawtooth crash. During the crash phase, the movement of the plasma core reaches the $q=1$ surface and the emissivity (mainly tungsten) drops. It should be noted that the emissivity is modulated before the crash due to the mode rotation and the accumulation of tungsten on the low-field side which is caused by centrifugal forces.

The effect of the crashes on the fast ions is illustrated in figure 13. Panel a shows the measured count rate of neutrons, mainly produced by fusion reactions between fast ions and the background plasma. It should be noted that the experimental neutron rate in arbitrary units has been measured by a novel neutron spectrometer [31] that has a very good signal to noise ratio when integrating the measurement in energy but does not provide absolute fluxes. Clearly, the neutron rate drops periodically at every sawtooth crash. The same behavior can be seen in the predicted neutron rates from TRANSP that are plotted in red. The TRANSP code models the effect of sawtooth crashes on the fast ions by applying the Kadomtsev model [32], which assumes full reconnection of the helical magnetic field due to the crash and redistributes fast particles according to the evolving field lines. TRANSP predicts an outwards fast-ion redistribution to a 

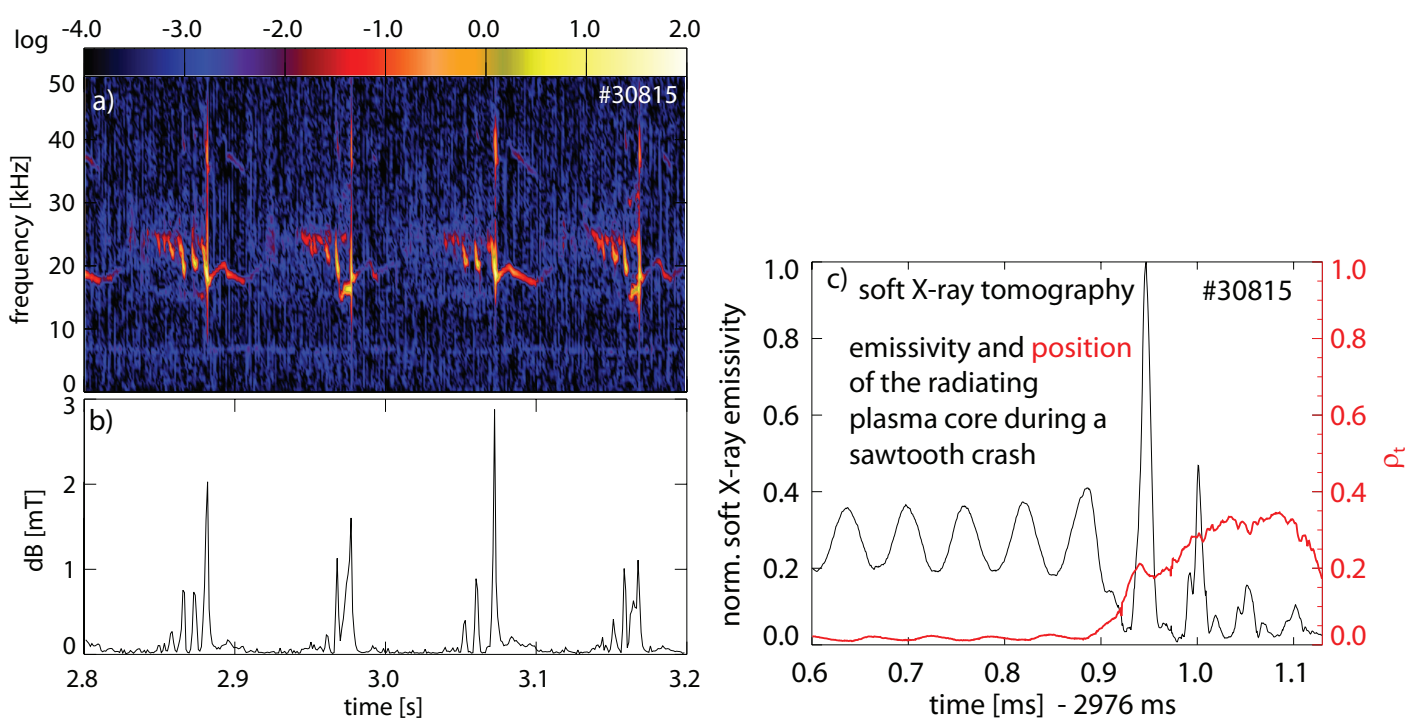

Figure 12. a) Magnetic spectrogram and amplitude of the magnetic perturbation (b). c) Emissivity and position of soft X-ray radiation during a sawtooth crash inferred by a tomographic reconstruction.

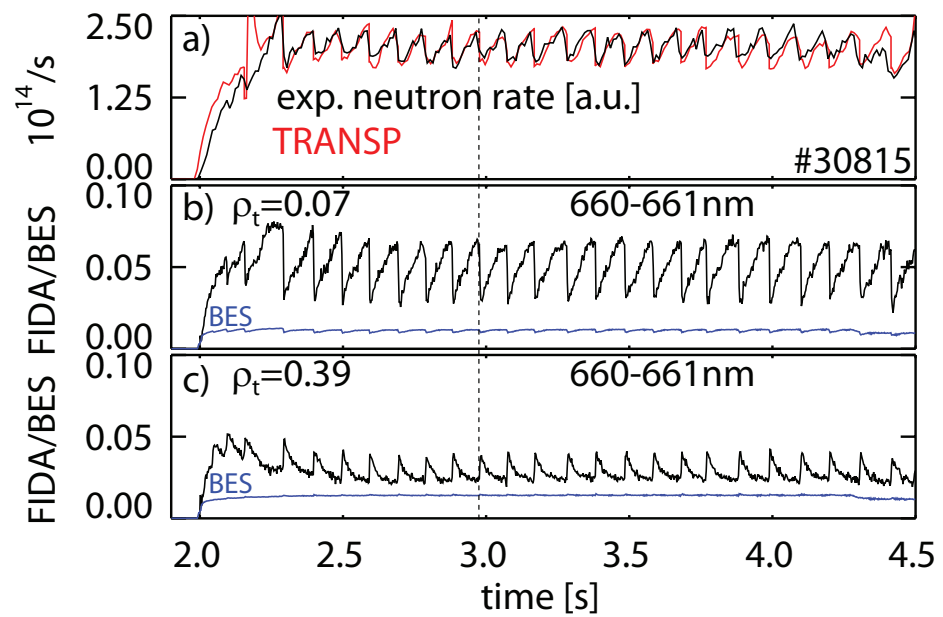

Figure 13. a) Measured neutron rate in arbitrary units, scaled to roughly match the prediction from TRANSP (Kadomtsev model) given in red. b) Temporal evolution of the measured FIDA/BES ratio at $\rho_{\mathrm{t}}=0.07$ showing a clear reduction of the fast-ion density when sawteeth appear. c) FIDA/BES ratio measured at $\rho_{\mathrm{t}}=0.39$, indicating an increased fast-ion density after sawtooth crashes outside the sawtooth inversion radius. In blue, the evolution of the beam emission is given in $2 \times 10^{19} \mathrm{photons} / \mathrm{s} / \mathrm{sr} / \mathrm{m}^{2}$ which has been used to normalize the FIDA data. 


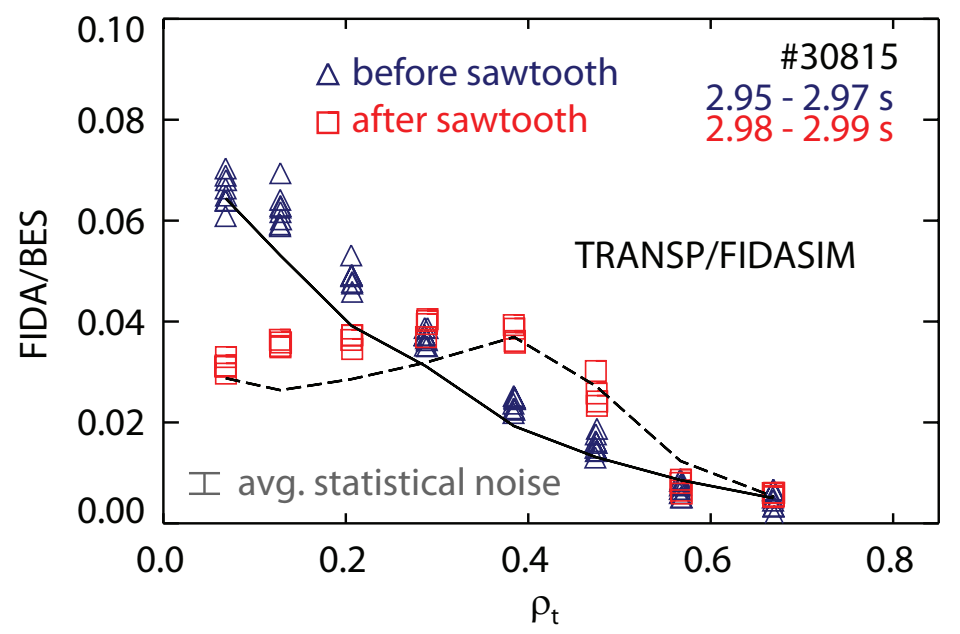

Figure 14. Radial FIDA/BES profiles $(660-661 \mathrm{~nm})$ acquired with a time resolution of $2.5 \mathrm{~ms}$ before (blue) and after (red) a sawtooth crash at $\approx 3 \mathrm{~s}$. In black, synthetic profiles from TRANSP+FIDASIM are given. The error-bar plotted in the lower left part of the figure illustrates the average statistical uncertainties of the FIDA measurement.

colder plasma region where fast ions are slowed down more quickly and hence contribute less to the neutron rate. The predicted relative change of the neutron rate in figure 13a agrees very well with the relative change in the experimental data. This suggests that the Kadomtsev model describes the sawtooth-induced fast-ion redistribution well. However, it should be noted that the neutron fluxes are dominated by specific parts of the fast-ion phase space [33] and no strong and global statement can be made here.

In panels $13 \mathrm{~b}$ and $13 \mathrm{c}$ we show the evolution of the integrated $(660-661 \mathrm{~nm})$ and normalized FIDA radiation observed by two toroidal lines of sight. The beam emission used to normalize the data is plotted in blue in figures $13 \mathrm{~b}$ and $13 \mathrm{c}$ and changes only weakly when sawtooth crashes appear. This shows that the probability of fast-ions to undergo charge exchange reactions and to emit FIDA radiation is not significantly modified. The strong drops of the central FIDA/BES ratio (figure 13b) and the increased signal outside the $q=1$ surface (figure $13 \mathrm{c}$ ) can, hence, be attributed to a radial fast-ion redistribution.

Figure 14 shows radial FIDA/BES profiles from the toroidal lines of sight before and after the sawtooth crash at $\approx 3 \mathrm{~s}$ as a function of $\rho_{\mathrm{t}}$. A constant offset has been subtracted from each channel to account for passive radiation from the plasma edge. The simulated FIDA/BES profiles, illustrated by the black lines, have been calculated by FIDASIM and the predicted change of the profiles is in good agreement with the measurement. This indicates that the sawtooth crashes cause an internal fast-ion 
redistribution of co-rotating fast-ions (observed by toroidal lines of sight between 660 and $661 \mathrm{~nm}$ ) that is dominated by the motion of fast particles along the evolving field line, as assumed in the Kadomtsev model.

In order to study the effect of sawtooth crashes on the central fast-ion velocity space distribution of fast ions, a tomographic inversion has been performed which has been demonstrated previously $[34,35,36]$ and is applied here to measure 2D fast-ion velocity distribution functions as a function of energy and pitch (pitch $=\mathrm{v}_{\|} / \mathrm{v}_{\text {tot }}$ where $\mathrm{v}_{\|}$is the fast-ion velocity anti-parallel to the magnetic field and $\mathrm{v}_{\text {tot }}$ is the total fast-ion velocity). The inversion is based on the analysis of FIDA spectra from four viewing geometries, shown in figure 1 in red. The four lines of sight intersect the path of NBI Q3 in the plasma center $\left(\rho_{\mathrm{t}}<0.09\right)$ and have angles to the local magnetic field of 12.4 degrees, 133.3 degrees, 68.7 degrees and 103.2 degrees. Depending on this observation angle and a given wavelength, each view collects FIDA radiation of neutralized fastions from different regions in velocity space, described by FIDA weight functions [37]. As an example, the toroidal view (12.4 degree) is sensitive to co-rotating fast-ions when analyzing strongly red-shifted FIDA light while the poloidal view (68.7 degree) is sensitive to fast-ions with pitch values close to zero at the blue shifted side.

Coherently averaged spectra from the four views, just before and just after sawtooth crashes, are plotted in figure 15 (coherent averaging has been used between $2.3 \mathrm{~s}$ and $4.5 \mathrm{~s}$ in discharge \#30815 to reduce the statistical uncertainties of the measurement). Not all wavelength regions can be analyzed because additional spectral components are present, in addition to the FIDA radiation. In particular, the cold D-alpha radiation at $656.1 \mathrm{~nm}$ (here blocked by a filter), the beam emission and strong impurity line emissions must be excluded from the analysis. Moreover, when performing tomographic inversions we use only FIDA light with Doppler shifts that correspond to fast ions with energies above $10 \mathrm{keV}$. In consequence, only the highlighted parts of the spectra (plotted in blue and red in figure 15) can be analyzed. Clearly, the measurement of the toroidal view (12.4 degree) shows a sawtooth-induced reduction of the FIDA radiation at red-shifted wavelengths while the poloidal view exhibits only a very weak variation at the blue shifted side. This already indicates that mainly co-rotating fast-ions are affected by the sawtooth crashes.

For a more detailed analysis that includes all accessible wavelength ranges and viewing geometries, a tomographic inversion has been applied that is based on a truncated singular value decomposition as in previous work [34, 35, 36]. In this method the forward model to calculate FIDA spectra is formulated in the form of a matrix 


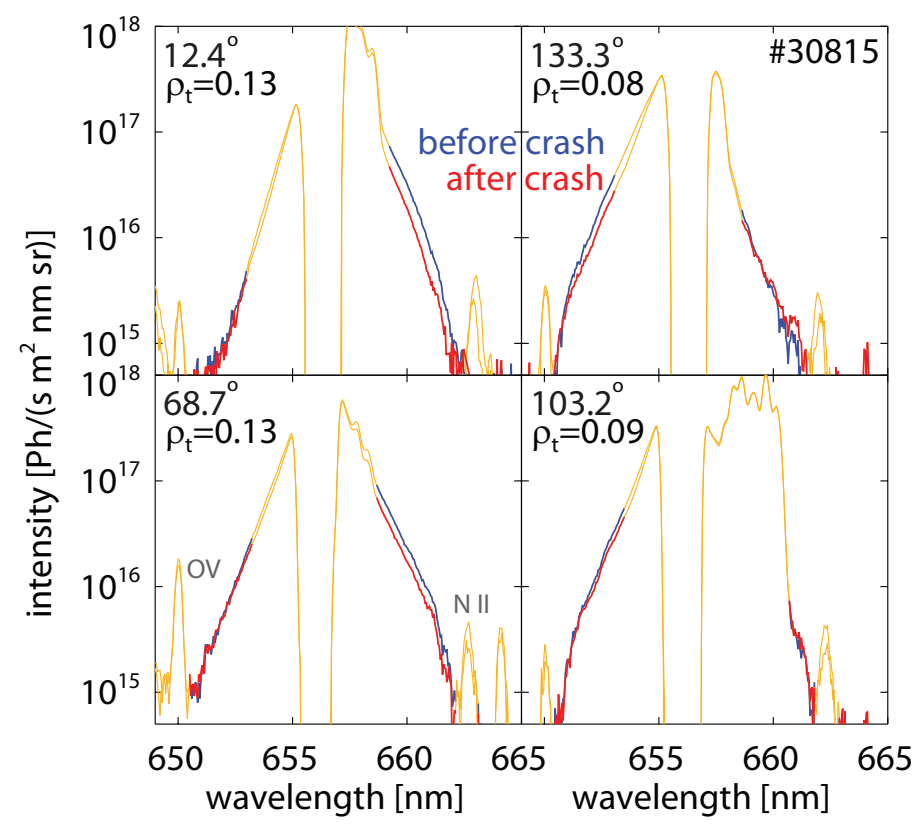

Figure 15. Time coherent averaged spectra containing FIDA radiation before (blue) and after (red) a sawtooth crash from four different viewing geometries on a semi logarithmic scale. The FIDA radiation can mainly be analyzed between 650 and $653 \mathrm{~nm}$ and between 659 and $662 \mathrm{~nm}$ where it is not superimposed by other spectral contributions, like the cold D-alpha radiation, the beam emission and impurity line emissions. The measured radiation plotted in yellow corresponds to the spectral regions that have been excluded from the tomographic reconstruction.

equation composed of weight functions which have been calculated by FIDASIM. The Moore-Penrose pseudoinverse of this transfer matrix is then calculated by a truncated singular value decomposition. Here we discretized the (energy,pitch)-space uniformly using $15 \times 15$ grid points for pitches from -1 to 1 and energies from 0 to $100 \mathrm{keV}$ and a truncation level of 38 has been applied. The inferred 2D fast-ion velocity distribution functions just before and just after the coherently averaged sawtooth crash are shown in figures $16 \mathrm{a}$ and 16b, respectively. The influence of the sawtooth crashes on the kinetic profiles and, hence, on the charge exchange and photon emission probability of fast ions is contained in the weight functions and, therefore, is considered by the tomography. As indicated by the cross-hatched area, we do not provide information on the low energy region because of our restriction in the observable wavelength range to fast ions above $10 \mathrm{keV}$.

The overall shapes of the reconstructed fast-ion distribution functions agree well with the fast-ion distribution functions predicted by TRANSP, shown in figures 16c and 16d. The simulations and the reconstructions before and after the crash show 

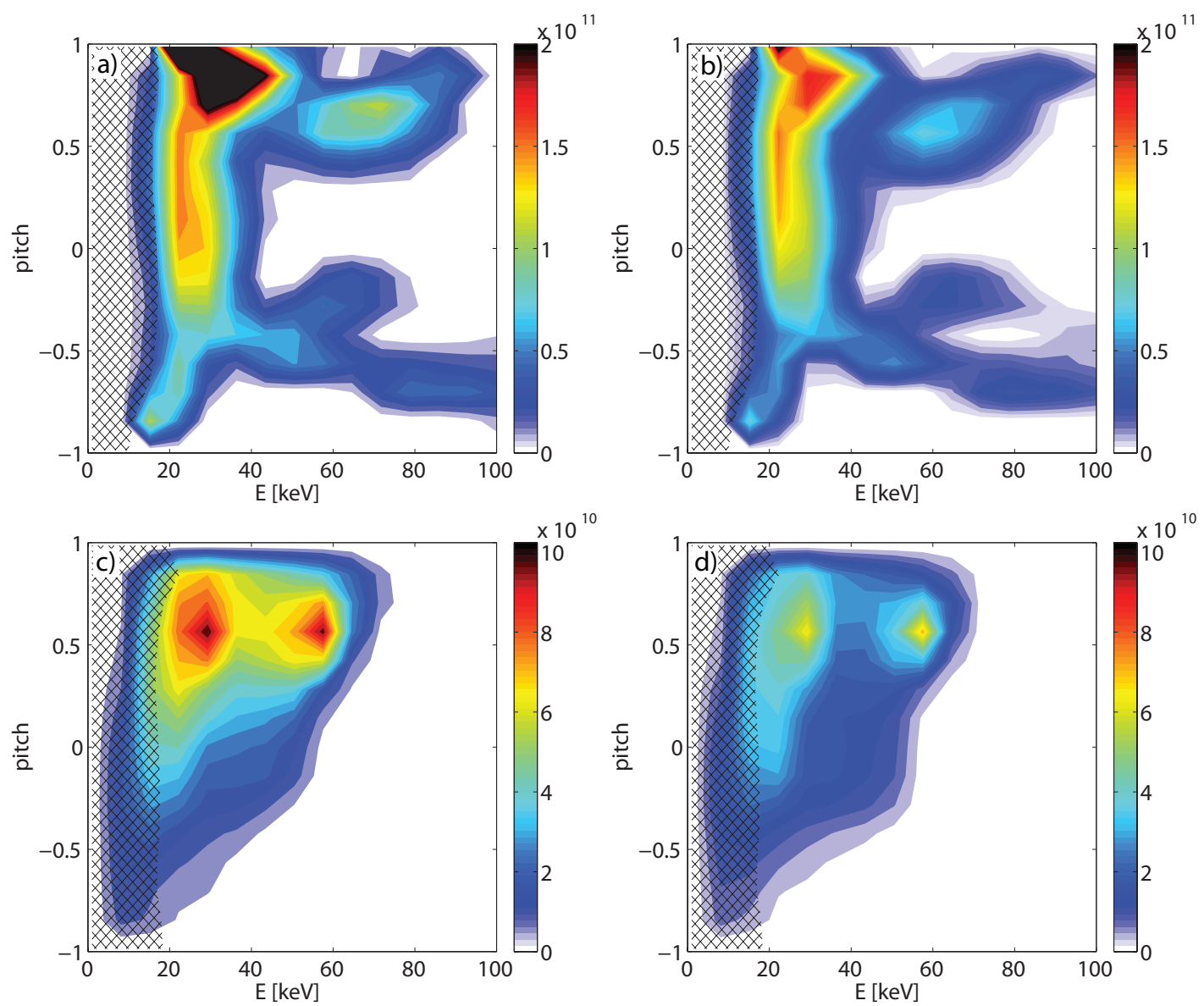

Figure 16. $a+b)$ Reconstructions of the fast-ion velocity-space distribution function in the plasma center (inside $\mathrm{q}=1$ ) before and after a sawtooth crash in units of [ions $\left.\left./\left(\mathrm{keVcm}^{3}\right)\right] . \mathrm{b}+\mathrm{c}\right)$ TRANSP predicted fast-ion velocity-space distribution function in the plasma center.

larger values for positive pitches than for negative pitches, explained by the co-current NBI injection geometry. Further, the beam injection peaks at full, half, and third injection energies $(60 \mathrm{keV}, 30 \mathrm{keV}$ and $20 \mathrm{keV})$ partly appear in the reconstructions. However, there are also some discrepancies possibly explained as we do not necessarily expect the TRANSP simulation to match the reconstruction since the discharge is not MHD quiescent but has strong $(1,1)$ activity also before the sawtooth crashes. Further, perfect subtraction of passive impurity radiation was not possible, which can also cause discrepancies: The reconstructions suggest slightly higher beam injection energy than expected from this $60 \mathrm{keV}$ source. We also observe non-zero fast-ion phasespace densities at energies very much higher than $60 \mathrm{keV}$ for positive as well as for negative pitches. These are likely artifacts of the reconstructions since similar features also appear in tomographic inversions of synthetic FIDA data for various truncation 


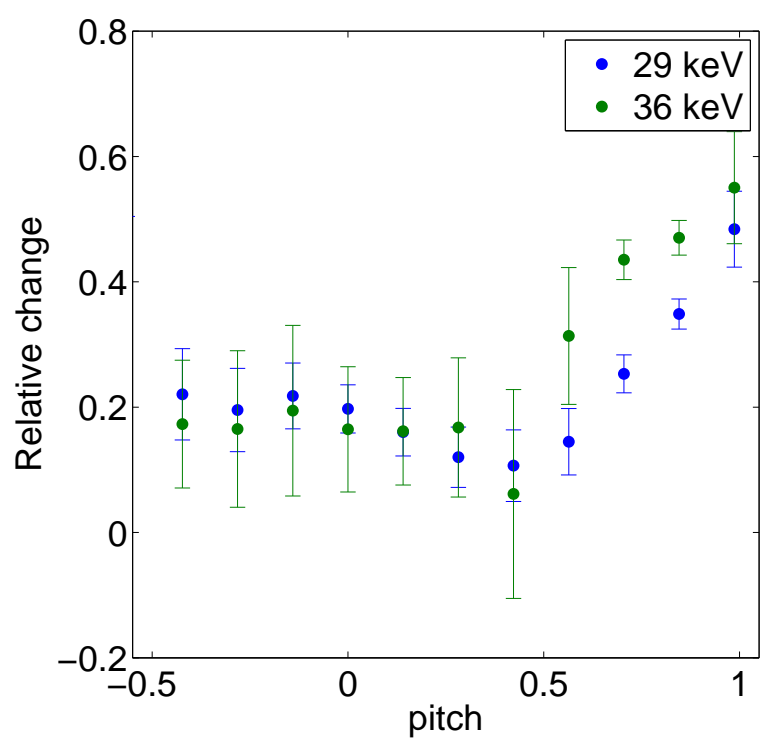

Figure 17. Relative change $\left(f_{\text {before }}-f_{\text {after }}\right) / f_{\text {before }}$ of the fast-ion density at $29 \mathrm{keV}$. and $36 \mathrm{keV}$ as a function of the pitch value. The error-bars represent the photon noise in the FIDA spectra used for the tomographic reconstruction.

levels of the singular value decomposition. With the experimental FIDA data, these artifacts are even stronger because we cannot measure the absence of FIDA light at large wavelength shifts when these wavelength ranges contain impurity line emissions. It is also possible that we have misleadingly identified weak impurity radiation at large Doppler shifts as FIDA radiation which could also lead to such tails. Lastly, it should be noted that we cannot distinguish between fast ions generated by NBI and the highenergy tail of the thermal distribution. Hence the tomographic reconstruction shows the sum of the fast ions that have been injected and the ions of the thermal distribution that have more energy than $10 \mathrm{keV}$.

Despite these uncertainties, the tomographic reconstruction allows us to provide, for the first time, a direct measurement of the redistribution level of fast ions. By integrating the inferred velocity space distribution in energy and pitch, we find a fast-ion density before the crash of $8.1 \times 10^{12}$ fast ions $/ \mathrm{cm}^{3}$ and a fast-ion density of $5.9 \times 10^{12}$ fast ions $/ \mathrm{cm}^{3}$ after the sawtooth crash. This corresponds to a redistribution of about $30 \%$ of the central fast ion population. It should be emphasized that we present an estimate covering the entire velocity-space above $10 \mathrm{keV}$ while previous studies $[6,9]$ reported on fast-ion densities only in specific parts of the velocity-space. In addition, the tomographic reconstruction permits us to study the pitch-angle dependence of the sawtooth-induced fast-ion redistribution. 
Figure 16c shows the relative changes in the fast-ion distribution function over the average sawtooth crash at $29 \mathrm{keV}$ and $36 \mathrm{keV}$ for pitches ranging from -0.5 to 1 . Here we focus on energies and pitches for which the amplitudes of the distribution functions are large and smooth. As can be seen, the relative change is strongly dependent on pitch. The changes are modest for small pitches up to 0.5 (5\% to $25 \%$ of redistribution) and strong for co-rotating ions with pitches close to 1 (up to $60 \%$ redistribution). The errorbars provided here represent the photon noise in the analyzed spectra and have been calcualted by the square roots of the diagonal elements of the covariance matrix of the inversion. It should be noted that the errorbars do not consider uncertainties in the weight functions or in the reconstruction algorithm.

The reconstruction shows that, particles moving parallel to the evolving field lines are affected most by sawtooth crashes while fast ions with significant velocity components perpendicular to the magnetic field are less sensitive to the sawtooth instability. This result is in agreement with [6] and [7] and will be compared with MHD modeling results in future publications. Moreover, it should be noted that further improvements of the tomographic reconstruction are planned by using a fifth FIDA view, by modeling or avoiding impurity radiation and by combining the FIDA measurements with data from collective Thomson scattering [38].

\section{Summary and conclusion}

In NBI current drive experiments, the loop-voltage changes in agreement with the neoclassical prediction when replacing on-axis NBI by off-axis NBI. However, measurements of the MSE diagnostic do not agree with the predictions by TRANSP. The possibility to explain this discrepancy with an increased fast-ion transport has been studied by analyzing radial profiles from a FIDA diagnostic. Here, measurements during on-axis NBI are in good agreement with the neo-classical prediction and during off-axis, the measured profiles are only slightly flatter than expected. The level of anomalous fastion diffusivity need to match the slightly flatter profiles cannot explain the discrepancy between the MSE measurement and the modeling. Possibly, $(1,1)$ MHD activity present in the discharges affects the plasma current distribution which is very difficult to consider in the simulation. Therefore, new experiments without $(1,1)$ MHD activity are planned in the 2015 experimental campaign, in particular aiming at experiment with elevated q-profiles.

During sawtooth crashes, very strong fast-ion redistribution is measured by an 
array of toroidal FIDA views and good agreement with the Kadomtsev model is found. 2D fast-ion velocity distribution functions in the plasma center have been calculated based on 4-view FIDA measurements which are easier to interpret than the raw FIDA spectra. They show a clear drop of the central fast-ion density after sawtooth crashes and a stronger redistribution of purely passing fast ions than of those ions with pitch values in the vicinity of zero.

\section{Acknowledgements}

This work has been carried out within the framework of the EUROfusion Consortium and has received funding from the Euratom research and training programme 20142018 under grant agreement No 633053. The views and opinions expressed herein do not necessarily reflect those of the European Commission.

[1] FISCH, N. J., Rev. Mod. Phys. 59 (1987) 175.

[2] EDITORS, I. P. B. et al., Nuclear Fusion 39 (1999) 2137.

[3] VON GOELER, S. et al., Phys. Rev. Lett. 33 (1974) 1201.

[4] MARCUS, F. B. et al., Plasma Physics and Controlled Fusion 33 (1991) 277.

[5] LOvBerG, J. A. et al., Physics of Fluids B: Plasma Physics 1 (1989) 874.

[6] Muscatello, C. M. et al., Plasma Physics and Controlled Fusion 54 (2012) 025006.

[7] NIELSEN, S. K. et al., Plasma Physics and Controlled Fusion 52 (2010) 092001.

[8] CECCOnello, M. et al., Plasma Physics and Controlled Fusion 57 (2015) 014006.

[9] GEIGER, B. et al., Nuclear Fusion 54 (2014) 022005.

[10] GUENTER, S. et al., Nuclear Fusion 47 (2007) 920.

[11] PARK, J. M. et al., Physics of Plasmas (1994-present) 16 (2009) .

[12] PACE, D. C. et al., Physics of Plasmas 20 (2013) 056108.

[13] GEIGER, B. et al., Plasma Physics and Controlled Fusion 57 (2015) 014018.

[14] A Herrmann, O. G. et al., Fusion Science and Technology 44 (2003) 569.

[15] HEIDBRINK, W. W. et al., Plasma Physics and Controlled Fusion 46 (2004) 1855.

[16] ZOHM, H. et al., Nuclear Fusion 41 (2001) 197.

[17] MÜCK, A. et al., Plasma Physics and Controlled Fusion 47 (2005) 1633.

[18] ISLER, R. C., Plasma Physics and Controlled Fusion 36 (1994) 171.

[19] CHEN, L. et al., Phys. Rev. Lett. 52 (1984) 1122.

[20] FISCHER, R. et al., Fusion Science and Technology 58 (2010) 675.

[21] GEIGER, B. et al., Review of Scientific Instruments 84 (2013) .

[22] HEIDBRINK, W. et al., Commun. Comput. Phys. 10 (2011) 716.

[23] HAWRYLUK, R. et al., in Physics of Plasmas Close to Thermonuclear Conditions 1 (1980) 19.

[24] PANKIN, A. et al., Computer Physics Communications 159 (2004) 157.

[25] SAUTER, O. et al., Physics of Plasmas (1994-present) 6 (1999) 2834.

[26] WESTERHOF, E., Rijnhuizen Report (1989) 89.

[27] LIN-LIU, Y. R. et al., Physics of Plasmas (1994-present) 4 (1997) 4179.

[28] IDA, K., Plasma Physics and Controlled Fusion 40 (1998) 1429. 
[29] HONDA, M. et al., Nuclear Fusion 52 (2012) 023021.

[30] IGOCHINE, V. et al., Nuclear Fusion 47 (2007) 23.

[31] TARDINI, G. et al., Journal of Instrumentation 7 (2012) C03004.

[32] PORCELli, F. et al., Plasma Physics and Controlled Fusion 38 (1996) 2163.

[33] JACOBSEN, A. et al., Nuclear Fusion 55 (2015) 053013.

[34] SALEWSKI, M. et al., Nuclear Fusion 52 (2012) 103008.

[35] SALEWSKI, M. et al., Nuclear Fusion 53 (2013) 063019.

[36] SALEWSKI, M. et al., Nuclear Fusion 54 (2014) 023005.

[37] SALEWSKI, M. et al., Plasma Physics and Controlled Fusion 56 (2014) 105005.

[38] NIELSEN, S. K. et al., Plasma Physics and Controlled Fusion 57 (2015) 035009. 